\title{
Percepção do usuário de comunicação suplementar ealternativa e de seus interlocutores sobre o uso dos sistemas gráficos
}

\section{Augmentative and alternative communication users and their interlocutors impressions on using graphic symbol system}

\author{
Maria Madalena Moraes Sant'Anna ${ }^{1}$, Debora Deliberato ${ }^{2}$, \\ Aila Narene Dahwache Criado Rocha ${ }^{3}$
}

http://dx.doi.org/10.11606/issn.2238-6149.v27i3p322-328

\begin{abstract}
Sant'Anna MMM, Deliberato D, Aila Narene Dahwache Criado Rocha ANDC. Percepção do usuário de comunicação suplementar e alternativa e de seus interlocutores sobre o uso dos sistemas gráficos. Rev Ter Ocup Univ São Paulo. 2016 set.-dez.;27(3):322-8.

RESUMO: Sistemas gráficos inseridos nos recursos de Comunicação Suplementar Alternativa (CSA) são ferramentas prioritárias para ampliar as possibilidades de comunicação. Objetivo: Descrever o histórico de uso da CSA de uma usuária, identificando a sua percepção e de seus interlocutores sobre os meios de comunicação utilizados. Procedimentos Metodológicos: Estudo de caso único, realizado por meio da análise dos dados obtidos do histórico da usuária de CSA nas intervenções de terapia ocupacional; duas entrevistas com a usuária e nove entrevistas com seus interlocutores. Resultados: Foram identificadas a importância da CSA para a facilitação da comunicação da usuária; a necessidade de reorganização constante dos símbolos em função da habilidade motora da usuária, bem como a relação entre a comunicação estabelecida e a demanda de suas atividades, sugerindo que a comunicação restrita com os interlocutores relacionar-se-ia com a pouca vivência de atividades sociais. Conclusões: Necessidade de uma reorganização das pranchas, envolvendo maior conteúdo de vocabulário, tornando a comunicação mais rápida; intervenções em sua rotina a fim de ampliar suas relações com seus interlocutores.
\end{abstract}

DESCRITORES: Educação especial; Paralisia cerebral; Pessoas com deficiência; Terapia ocupacional.
Sant'Anna MMM, Deliberato D, Aila Narene Dahwache Criado Rocha ANDC. Augmentative and alternative communication users and their interlocutors impressions on using graphic symbol system. Rev Ter Ocup Univ São Paulo. 2016 Sept.Dec.;27(3):322-8.

ABSTRACT: Graphic symbol systems used in Augmentative and
Alternative Communication (AAC) are priority tools to expand
communication possibilities. Objective: Describe the use of
AAC by a female user, characterizing her and her interlocutors
impressions on the means of communication used. Methods:
Single case study, analyzing data obtained from AAC user history
in occupational therapy interventions; two interviews with the user
and nine interviews with her interlocutors. Results: We recognized
AAC importance to facilitate user communication; the necessity
of constantly rearranging symbols due to user motor skills; and
the relationship between the established communication and
activities demand, suggesting that limited communication with
her interlocutors would be related to lack of social interaction.
Conclusion: It was necessary to rearrange the boards, include
greater vocabulary content to make communication faster;
interventions in her routine to expand her relationship with her
interlocutors. KEYWORDS: Special education; Cerebral palsy; Disabled individuals; Occupational therapy.

Estudo apresentado na disciplina de Comunicação Alternativa e Suplementar: Recursos e Procedimentos Utilizados na Educação do Programa de Pós-Graduação em Educação, linha Educação Especial, UNESP, Marília (SP).

1. Doutora em Educação pelo Programa de Pós-graduação em Educação - UNESP/Marília; Coordenadora Pedagógica da Pós-Graduação

Lato Sensu Terapia Ocupacional: Uma Visão Dinâmica em Neurologia, FAMESP(SP).

2. Professora Adjunta do Departamento de Educação Especial e do Programa de Pós-Graduação em Educação da UNESP/Marília.

3. Doutora em Educação pelo Programa de Pós Graduação em Educação - UNESP/Marília; Professora Assistente do Curso de Terapia Ocupacional, Departamento de Fisioterapia e Terapia ocupacional da UNESP/Marília.

Endereço para Correspondência: Maria Madalena Moraes Sant'Anna. Avenida Garibaldi Deliberador nº 545 - Bloco, 1 apto. 34 . CEP. 86050-280 - Londrina, SP. Email: madasantanna2@gmail.com 


\section{INTRODUÇÃO}

comunicação humana nutre as relações
humanas e envolve a disponibilidade de
identificar parceiros de comunicação para compartilhar desejos, opiniões, vontades, inclusive da pessoa com deficiência apresentando severas dificuldades motoras e que utiliza um sistema gráfico para se comunicar ${ }^{1}$.

A pessoa com deficiência física, com dificuldade de oralização, pode usar a Comunicação Suplementar e Alternativa (CSA) por meio de diferentes recursos, para viabilizar sua comunicação social propiciando a construção de suas redes interpessoais ${ }^{2}$.

A CSA envolve recursos, sistemas, técnicas e serviços para que pessoas com dificuldades na comunicação sejam capacitadas a estabelecer diálogos e obter autonomia em suas atividades da vida diária. As pranchas com figuras, símbolos, letras, palavras e o computador, são considerados os recursos mais frequentes entre usuários de sistemas ${ }^{3}$.

A American Speech-Language-Heraring Association (ASHA) definiu que a CSA envolve clínica, educação e pesquisa, atuando nas incapacidades de pessoas com distúrbios da comunicação expressiva, gestual, oral ou escrita e/ou distúrbios da compreensão ${ }^{4}$.

No Brasil, a CSA iniciou-se no final da década de 70, por meio de sistemas gráficos, com os esforços de alguns profissionais em parceria com alguns pais, em São Paulo, Rio de Janeiro, Minas Gerais e Rio Grande do Sul, sendo o sistema Bliss o mais utilizado ${ }^{5,6,7}$.

Propor uma linguagem por intermédio da CSA não é apenas estabelecer a implantação de símbolos, mas garantir que esta se desenvolva e seja aplicada em todos os meios sociais. Assim, ampliar o seu uso é a meta do terapeuta ocupacional em seus procedimentos clínicos juntamente com a equipe envolvida nas intervenções, e com a família ${ }^{8,9}$.

Em estudo de caso único realizado com pais de crianças com dificuldade de comunicação, foram apontados os obstáculos na utilização dos sistemas de comunicação, pelos interlocutores, e a necessidade premente de educar a sociedade para permitir que um usuário construa parcerias de comunicação em seu dia a dia ${ }^{10}$.

Assim, este estudo de caso descreveu o histórico de uso da CSA de uma usuária, identificando a sua percepção e a de seus interlocutores sobre os meios da CSA utilizados em suas atividades do dia a dia.

\section{PROCEDIMENTOS METODOLÓGICOS}

Estudo de caso de uma jovem usuária da CSA. O desenho experimental de caso possui dois elementos fundamentais: avaliações repetidas e o desenho de fases. Justifica-se a opção metodológica, considerando-se a exploração de situações da vida real cujos limites não estão claramente explícitos ${ }^{11}$. Fez parte de um projeto maior a respeito das "Implementações de recursos de comunicação suplementar e alternativa no contexto escolar e familiar", aprovado no Comitê de Ética com o parecer n ${ }^{\circ}$ 1202/2006.

\section{Participantes}

Jovem identificada como Maria, 26 anos de idade, gênero feminino, usuária da CSA desde os seis anos, diagnosticada com paralisia cerebral, bem como nove pessoas consideradas seus principais interlocutores.

Cientes dos objetivos, todos assinaram o termo de consentimento livre e esclarecido.

\section{Coleta de dados}

Utilizaram-se múltiplas fontes de evidências, com triangulação dos dados, organizando-se uma base de dados com relatórios de evolução e avaliações clínicas selecionadas nas anotações do histórico das intervenções de terapia ocupacional com a participante, registros de áudio e audiovisuais das entrevistas com a usuária e interlocutores ${ }^{12}$.

Para complementar os dados, propôs-se a participação de observadores externos, ou seja, leitores do caso, para ampliar as evidências dos resultados e a conclusão deste estudo. Foram coletados dados com entrevista semiestruturada com dois roteiros prévios: usuária e interlocutores. Entrevistas semiestruturadas identificam dados e informações, apresentando, como característica, a necessidade de roteiro prévio ${ }^{13}$. Os roteiros foram enviados a um comitê de três juízes experts na área de CSA.

Para a fidedignidade das perguntas, realizou-se o teste de confiabilidade. Após a sua devolução, pelos juízes, foi elaborada uma versão e reencaminhada ao mesmo comitê. Posteriormente à aprovação, elaborou-se a versão final.

A entrevista com a usuária da CSA foi realizada em sua casa, gravada e filmada, envolvendo 11 questões:

1. Quais as formas de comunicação que você utiliza para ser compreendida pelas pessoas?

2. Você se lembra de como foi começar a usar a prancha para se comunicar?

3. A prancha sempre foi mais ou menos desse jeito ou passou por muitas mudanças. Quais? 
4. Com quais pessoas você se comunica utilizando a prancha?

5. Com quem você tem mais facilidade para se comunicar e, geralmente, vocês conversam sobre o quê?

6. Você tem alguma atividade fora de casa. Qual?

7. Como você se comunica com as pessoas lá?

8. Sobre o que conversam?

9. Você acha que as pessoas nesses lugares têm dificuldade de conversar com você?

10. Você acha que a prancha de comunicação que você usa está boa o suficiente para ajudar a conversar com as pessoas?

11. Você acha que poderia melhorar?

Transcreveu-se a entrevista com correções gramaticais. Após a análise de conteúdo ${ }^{14}$, identificaramse três categorias de interlocutores: semanal, quinzenal e esporádico. Novo contato foi mantido com a usuária (filmado e gravado), para confirmar seus interlocutores e a respectiva frequência de contato.

Os interlocutores semanais foram avó materna, mãe, prima, irmã, madrinha; o cunhado era interlocutor quinzenal, e, esporadicamente, a usuária se comunicava com padrinho, amiga, prima da mãe e pai.

Dos dez interlocutores identificados, nove foram entrevistados. Dentre eles, oito conversavam com Maria por meio da prancha e um por computador. As entrevistas com os interlocutores foram gravadas e realizadas com roteiro contendo cinco questões:

1. Como você se comunica com Maria e com que frequência?

2. Sobre o que vocês costumam conversar?

3. O que você acha sobre essa forma de se comunicar com Maria?

4. Vocês realizam alguma atividade social, juntos? Com que frequência? Como é a comunicação dela nesse local?

5. Você tem alguma sugestão do que poderia ajudá-la a se comunicar melhor?

Após estas entrevistas, o material obtido em áudio foi transcrito com correções gramaticais.

\section{Análise dos dados}

A partir dos dados obtidos do histórico da usuária de CSA, na terapia ocupacional e em entrevistas com a usuária e com os interlocutores, realizou-se a triangulação dos dados ${ }^{12}$, organizando-se um documento único temporal e com identificação da fonte Arial.

Finalizado o documento, procedeu-se a análise de conteúdo ${ }^{14}$ sendo identificadas duas categorias: o percurso e a percepção de Maria no uso da CSA; a percepção de seus interlocutores sobre o uso da CSA em seu dia a dia.

\section{RESULTADOS E DISCUSSÕES}

Maria nasceu em 28/2/1989 com quadro de paralisia cerebraldiscinética ouatetóide,commaiorcomprometimento à direita. Utiliza como meio de locomoção uma cadeira de rodas manual, conduzida por outra pessoa. O Gross Motor Function Measure Classification System ${ }^{15}$ (GMFCS) foi definido como nível $\mathrm{V}$, aos 18 anos de idade, ou seja, todas as suas áreas de função motora estavam limitadas e o uso da tecnologia assistiva era restrito, permanecendo na idade atual com as mesmas características motoras. Como meio de comunicação, utiliza prancha com alfabetos, números e palavras.

Iniciou seu percurso escolar em 1991 em escola especial; em 1993 frequentou sala especial em escola regular; em 1994, aos quatro anos de idade, sala comum da educação infantil e, a partir desta etapa, cursou escola regular com acompanhamento de tutora, finalizando o ensino médio aos 17 anos, quando interrompeu seus estudos.

Em 1992, terapeuta ocupacional e fonoaudióloga encarregaram-se de organizar figuras que Maria já usava, selecionando, por tema, 20 figuras relevantes em sua rotina. Elaborou-se uma prancha para a escola com essas figuras, quando Maria desejava comunicar-se, indicava-as com o dedo mínimo da mão esquerda.

Na sequência, em 1994, iniciou-se o uso de símbolos da comunicação pictórica (do inglês Picture Communication Symbols - PCS $)^{16}$ e do Bliss ${ }^{7}$. As informações sobre esses sistemas eram escassas no Brasil, dificultando o seu uso, para os profissionais e para as famílias.

Prosseguiu-se seguindo o padrão de cores do $\mathrm{PCS}^{16}$, com prancha em formato de pasta com figuras representativas (eu, mamãe, filha, vou, casa, escola), facilitando a sua comunicação, complementada com o "sim" e o "não", expressos com gesto de cabeça.

Inicialmente, optou-se por incentivar Maria a responder às perguntas como "Quem levou você para a escola?" E, aos seis anos de idade, apontava o símbolo da mamãe; o verbo levar correspondia ao símbolo da escola. A forma de apontar era sempre com o dedo mínimo da mão esquerda. Seguindo os padrões formais de construção, chegou-se à primeira prancha com 162 símbolos, no período dos 6 aos 10 anos (Quadro 1). 
Sant'Anna MMM, et al. Percepção do usuário de comunicação suplementar. Rev Ter Ocup Univ São Paulo. 2016 set./dez.;27(3):322-8.

Quadro 1 - Descrição dos símbolos da Primeira Prancha

\begin{tabular}{|l|c|c|}
\hline Categorias & $\begin{array}{c}\text { Cor do fundo } \\
\text { dos símbolos }\end{array}$ & $\begin{array}{c}\text { Quantidade } \\
\text { de símbolos }\end{array}$ \\
\hline Pessoas & Amarelo & 19 \\
\hline Verbos & Verde & 35 \\
\hline Substantivos & Laranja claro & 48 \\
\hline Adjetivos & Azul & 8 \\
\hline $\begin{array}{l}\text { Formas de cumprimentar as } \\
\text { pessoas }\end{array}$ & Rosa & 7 \\
\hline $\begin{array}{l}\text { Vogais, números, alfabeto, } \\
\text { datas, perguntas }\end{array}$ & Branco & 45 \\
\hline Total de símbolos & $\mathbf{1 6 2}$ \\
\hline
\end{tabular}

Fonte: Elaborado pelos autores.

No mesmo formato, foi criada sua segunda prancha, para o período da adolescência e da juventude, com 398 símbolos (Quadro 2), emergindo a necessidade de pranchas para assuntos pontuais. Construíram-se três modelos utilizados conforme o ambiente em que Maria estava, uma específica para a aula de música, outra para atividades sociais e escola (Quadros 3, 4 e 5).

Quadro 2 - Descrição dos símbolos da Segunda Prancha

\begin{tabular}{|l|c|c|}
\hline Categorias & $\begin{array}{c}\text { Cor do fundo } \\
\text { dos símbolos }\end{array}$ & $\begin{array}{c}\text { Quantidade } \\
\text { de símbolos }\end{array}$ \\
\hline Verbos & Verde & 36 \\
\hline Substantivos & Laranja Claro & 145 \\
\hline Adjetivos & Azul & 46 \\
\hline $\begin{array}{l}\text { Formas de cumprimentar as } \\
\text { pessoas }\end{array}$ & Rosa & 15 \\
\hline $\begin{array}{l}\text { Vogais, números, alfabeto, } \\
\text { cores, dias da semana, } \\
\text { nomes }\end{array}$ & Branco & 156 \\
\hline Total de símbolos & $\mathbf{3 9 8}$ \\
\hline
\end{tabular}

Fonte: Elaborado pelos autores.

Quadro 3 - Prancha de apoio para a aula de música

\begin{tabular}{|l|l|c|}
\hline Categorias & $\begin{array}{l}\text { Cor do fundo dos } \\
\text { símbolos }\end{array}$ & $\begin{array}{c}\text { Quantidade } \\
\text { de símbolos }\end{array}$ \\
\hline Alfabeto & Branco & 27 \\
\hline Números & Branco & 10 \\
\hline Sinais ortográficos & Branco & 14 \\
\hline Fotografia & Colorido & 1 \\
\hline Notas e escala musical & Branco & 19 \\
\hline Total de símbolos & $\mathbf{7 1}$ \\
\hline
\end{tabular}

Fonte: Elaborado pelos autores.
Quadro 4 - Prancha de apoio para atividades sociais e escola

\begin{tabular}{|l|l|c|}
\hline Categorias & $\begin{array}{l}\text { Cor do fundo } \\
\text { dos símbolos }\end{array}$ & $\begin{array}{c}\text { Quantidade } \\
\text { de símbolos }\end{array}$ \\
\hline Pedidos e cumprimentos & Rosa & 20 \\
\hline $\begin{array}{l}\text { Preposições, calendário } \\
\text { e datas }\end{array}$ & Branco & 54 \\
\hline Pessoas & Amarelo & 24 \\
\hline Verbos & Verde & 68 \\
\hline Substantivos & Laranja & 39 \\
\hline Adjetivos & Azul claro & 76 \\
\hline Total de símbolos & & 281 \\
\hline
\end{tabular}

Fonte: Elaborado pelos autores.

Quadro 5 - Prancha de apoio da escola

\begin{tabular}{|l|l|c|}
\hline Categorias & $\begin{array}{l}\text { Cor do fundo } \\
\text { dos símbolos }\end{array}$ & $\begin{array}{c}\text { Quantidade de } \\
\text { símbolos }\end{array}$ \\
\hline Alfabeto & Branco & 26 \\
\hline Número & Branco & 10 \\
\hline Sinais ortográficos & Branco & 11 \\
\hline Total de símbolos & 47 \\
\hline
\end{tabular}

Fonte: Elaborado pelos autores.

Paralelamente, Maria tinha na mesa da cadeira de rodas uma prancha com 365 símbolos, com a mesma estrutura de categorias, nas quais o número de elementos diminuía conforme aumentava o domínio de leitura e escrita.

Percebeu-se que preferia usar o alfabeto para formar frases e estruturar seu diálogo. A prancha foi reduzida até atingir 37 símbolos representando o alfabeto, os números e 48 palavras (verbos, substantivos, pessoas, preposições, com a cor branca de fundo).

Em 1997, com oito anos de idade, Maria cursava o $2^{\circ}$ ano do Fundamental 1 do ensino regular e utilizou um vocalizador Voxtable com mensagens rápidas por curto período de tempo. A voz sintetizada masculina desagradava Maria, sendo um dos motivos da interrupção. Com o teclado Intelekey com colmeia e mouse adaptados, em 15 minutos, digitava frases como "Eu vou sita estra ai no consultório jogar".

Inicia, em 2014, o uso de Ipad posicionado em suporte fixo à mesa, manuseando-o com alguns dedos do membro superior esquerdo. A prancha portátil confeccionada em papel possui 85 símbolos que permitem a comunicação com as pessoas da família e amigos próximos. Sim e não são representados com gesto de cabeça e alguns sinais de libras. 
As comunicações mais constantes são com as pessoas da família, com maior frequência das pessoas que cuidam, respondendo a pedidos de ligar e desligar aparelhos (rádio), ir ao banheiro e, muitas vezes, com o olhar dirigido à prancha.

Os interlocutores foram unânimes quanto à importância da prancha de comunicação. Algumas vezes, Maria utiliza-se do sistema de varredura, olhando na direção dos símbolos, ou o interlocutor aponta um símbolo com o dedo, e Maria responde com "sim" ou "não" com a cabeça, outras vezes ela inicia uma palavra e, alguns interlocutores finalizam, e Maria concorda ou discorda com a cabeça. Os movimentos de Maria estão mais restritos devido às suas dificuldades motoras que vêm aumentando no membro superior, o que torna o sistema de varredura e abreviação das palavras mais viável.

A literatura identifica que, para os usuários da CSA com paralisia cerebral, a família representa a figura central para a adesão aos sistemas de comunicação, sendo parceiros importantes na intervenção, e que necessitam de treinamento constante, pois "as crianças não se tornarão usuários competentes de signos gestuais, gráficos e tangíveis sem que os membros da família compreendam e apoiem esse esforço" ${ }^{\prime 1}$.

Esta afirmação é ratificada nos momentos de comunicação de Maria com a mãe e a avó, correspondendo às conversas mais detalhadas, falando sobre o que deseja. Com a mãe, as atividades são mais frequentes como ir ao shopping, pequenas viagens, mercado. Algumas vezes, acompanha-a em palestras sobre o seu processo de comunicação, contando um pouco de sua história, a partir de solicitações da comunidade acadêmica de sua cidade. No sítio, a avó tenta manter uma vida campestre com Maria, vivenciando as atividades de rotina. Muitas pessoas frequentam o sítio e algumas têm disponibilidade de passar algum tempo com Maria conversando por meio da prancha.

Destaca-se a necessidade de oferecer capacitação de interlocutores nas diferentes habilidades comunicativas, para que criança e jovem com paralisia cerebral possam compartilhar seus desejos, conhecimentos com diferentes pessoas, nos diversos contextos ${ }^{18,19}$.

Três interlocutores, sugeriram que uma mudança na prancha, com a inclusão de mais palavras, facilitaria a comunicação; pois o processo de montar a palavra, a partir das letras, torna a conversa mais demorada, fazendo com que Maria expresse sua opinião com muita lentidão e, portanto, quando o assunto no grupo expirou; os interlocutores, por sua vez, desistem de esperar finalizando a frase por Maria. Um interlocutor aponta que, frequentemente, sente Maria "com preguiça", iniciando as frases, mas não terminando, esperando que as pessoas complementem ou adivinhem.

Nesta perspectiva, há necessidade da inserção da família no momento de avaliar, selecionar e inserir os sistemas gráficos com crianças e jovens com paralisia cerebral. Os membros da família são os principais interlocutores dos usuários de sistemas gráficos, e devem ser os informantes mais atentos quanto às demais habilidades expressivas utilizadas pelo jovem com deficiência ${ }^{8}$.

Quanto às atividades sociais, o seu final de semana e feriados no sítio foram os mais citados, onde Maria conversa com as pessoas da família e algumas atividades diferenciadas acontecem como as atividades culinárias, citadas por uma interlocutora, em que a função de Maria é a de amassar a massa. Um dos interlocutores menciona que Maria pergunta, às vezes, sobre alguém da família, espontaneamente. Outro assunto recorrente, apontado pela maioria, são os questionamentos às atividades que fez durante a semana. Disseram usar esse tema para facilitar o diálogo.

Os estudos na área da CSA destacam a necessidade de atenção aos diferentes parceiros em cada ambiente, às oportunidades de comunicação que dispensam ao indivíduo e o modo como interagem; pois, a principal característica das dificuldades de comunicação de indivíduos não oralizados, é a baixa expectativa que seus parceiros têm em relação à sua capacidade de veicular e produzir novas informações de forma complexa ${ }^{20,21}$.

Também foram citadas como atividades sociais, as datas festivas e temáticas, como Natal e aniversários de pessoas da família. Nestas ocasiões, Maria pede para ir ao shopping comprar presente. Um interlocutor afirmou que, esporadicamente, saem juntos para ir ao shopping.

Outro interlocutor relatou que a conversa é feita, sobretudo, com brincadeiras, gerando uma estrutura mais infantilizada que o esperado para a sua idade cronológica. Disse que, eventualmente, Maria permanece mais tempo no mesmo assunto. Percebe que, hoje, a sua comunicação diminuiu muito em relação à que tinha anteriormente, assim como diminuíram as suas atividades no dia a dia. A ausência de diversificação e a redução do número de interlocutores na sua rotina pode ser um dos fatores que justifique a regressão na qualidade da comunicação, isto é, a ruptura com os compromissos sociais comprometeu seu aprendizado adquirido, bem como sua evolução.

Considerando-se que as atividades sociais do usuário da CSA podem ser restritas, quando comparadas às pessoas oralizadas da mesma faixa etária, existe a 
preocupação com um conjunto de temas a respeito do uso da CSA na comunicação com interface da interação social, concebendo que o sucesso na implementação de determinado recurso ocorrerá quando se expandir a capacidade funcional do usuário de $\mathrm{CSA}^{3}$.

Em relação ao computador e demais tecnologias, Maria não as utiliza com frequência. O Ipad, quando usado, restringe-se à digitação, sempre respondendo às perguntas solicitadas.

A partir de seu domínio sobre o alfabeto, a conversa apresentou melhora significante, limitando-se, contudo, às respostas das perguntas relativas às solicitações com seus cuidados básicos. Maria passou a não manifestar iniciativa na comunicação, dificilmente elaborando perguntas e questionamentos.

A família e outros parceiros de comunicação, muitas vezes, são insensíveis às tentativas de comunicação com pessoas usuárias de CSA. Acabam dominando as interações comunicativas, procedendo de diversas maneiras que não as favorecem: fornecem poucas oportunidades de comunicação; abstêm-se de perguntas abertas, formalizando, apenas, questões de sim e não; interrompem o usuário quando está usando a CSA e, ainda, enfocam o recurso ou a estratégia de comunicação, ao invés de focar no usuário e na respectiva mensagem que deseja expressar ${ }^{22}$.

Três interlocutores foram claros em afirmar que, para ampliar a possibilidade de diálogos da usuária, é fundamental ter disponibilidade, paciência e desejo em compartilhar uma conversa com Maria.

Este estudo de caso possibilitou destacar que as intervenções junto aos usuários da CSA devem selecionar recursos adequados às suas necessidades,

\section{REFERÊNCIAS}

1. Von Tetzchner S. Suporte ao desenvolvimento da comunicação suplementar e alternativa. In: Deliberato D, Gonçalves MJ, Macedo EC. Comunicação alternativa: teoria, prática, tecnologias e pesquisa. São Paulo: Memnon; 2009. p.14-27.

2. Light JC. Development of communicative competence by individuals who use AAC. In: Light JC, Beukelman DR, Reichle, J. Communicative competence for individuals who use AAC: from research to effective practice. Baltimore (MD): Brookes; 2003. p.3-38. promover inclusão em ambientes frequentados por seus pares e capacitar diferentes parceiros de comunicação. É necessário, ainda, estimular o uso da CSA para potencializar as diferentes atividades da rotina do usuário.

\section{CONCLUSÃO}

A CSA, implantada por meio da prancha com palavras e letras, foi o recurso mais utilizado pela usuária em seu dia a dia e o que possibilitou a facilitação da comunicação e interação social.

Com a redução de suas atividades, bem como as dificuldades motoras e emocionais que Maria começou a apresentar nos últimos anos, seu tempo para a formação de palavras por meio do alfabeto aumentou, identificando-se que, com maior número de símbolos compostos por palavras em sua prancha, sua comunicação poderia ser mais rápida, facilitando e melhorando a interação com os interlocutores.

Este estudo de caso identificou nos relatos da usuária e de seus interlocutores, o efeito positivo da implantação do uso da CSA pelo terapeuta ocupacional e pelo fonoaudiólogo desde a infância da usuária, pois esta é a forma que possibilita o contato e o convívio com seus familiares.

Considera-se que o terapeuta ocupacional e o fonoaudiólogo envolvidos na implantação dos sistemas de CSA devem ter como prioridade, juntamente com a família, de investir no treinamento dos interlocutores para o uso diário dos recursos da CSA nas diferentes etapas do desenvolvimento da vida do usuário deste sistema de comunicação e, paralelamente, construir, junto com ele, possibilidades e rotinas significativas que viabilizem interlocutores disponíveis para a comunicação, garantindo o direito à verdadeira inclusão social. 
Sant’Anna MMM, et al. Percepção do usuário de comunicação suplementar. Rev Ter Ocup Univ São Paulo. 2016 set./dez:;27(3):322-8.

5. Chun RYS. O desenvolvimento da comunicação não verbal através dos símbolos Bliss em indivíduo não falante portador de paralisia cerebral. Distur Comun. 1991;4(2):121-36.

6. Sampaio VC, Pereira MFM. Comunicação alternativa. In: Cury VCR, Brandão MB. Reabilitação em paralisia cerebral. Rio de Janeiro: Med Book; 2011. p.264-82.

7. Blissymbolics Communication International (BCI). About Blissymbolics. 2015 [cited 2016 Jan 24]. Available from: $\quad$ http://www.blissymbolics.org/index.php/aboutblissymbolics.

8. Deliberato D, Manzini EJ. Identification of the communicative abilities of brazilian children with cerebral palsy in the family context. J Commun Disord Q. 2012;33:195-201. doi: 10.1177/1525740110384130.

9. Associação Americana de Terapia Ocupacional. Estrutura da prática da terapia ocupacional: domínio \& processo. Rev Ter Ocup Univ São Paulo. 2015;26(ed. esp.):1-49. Disponível em: http://www.revistas.usp.br/rto/article/ view/97496/96423.

10. McNaughton D, Rackensperger T, Benedek-Wood E, Krezman C, Williams MB, Light J. A child needs to be given a chance to succeed: parents of individuals who use AAC describe the benefits and challenges of learning AAC technologies. Augment Altern Commun. 2008;24(1):43-55. doi: $10.1080 / 07434610701421007$.

11. Gil AC. Como elaborar projetos de pesquisa. 4a ed. São Paulo: Atlas; 2002 [citado 24 fev. 2015]. Disponível em: https://professores.faccat.br/moodle/pluginfile.php/13410/ mod_resource/content/1/como_elaborar_projeto_de_ pesquisa_-_antonio_carlos_gil.pdf.

12. Triviños ANS. Introdução à pesquisa em ciências sociais: a pesquisa qualitativa em educação. São Paulo: Atlas; 1987 [citado 2015 mar 09]. Disponível em: http://pt.scribd.com/ doc/84708933/Livro-Introducao-a-pesquisa-em-CienciasSociais-Trivinos\#scribd.

13. Manzini EJ. Considerações sobre a elaboração de roteiro para entrevista semi-estruturada. In: Marquezine MC, Almeida MA, Omote S. Colóquios sobre pesquisa em educação especial. Londrina: Eduel; 2003. p.11-25 [citdo 30 abr. 2015]. Disponível em: http://www.marilia.
unesp.br/Home/Instituicao/Docentes/EduardoManzini/ Consideracoes_sobre_a_elaboracao_do_roteiro.pdf.

14. Bardin L. Análise de conteúdo. Lisboa: Edições 70; 2004.

15. Hiratuka E, Matsukura TS, Pfeifer LI. Adaptação transcultural para o Brasil do Sistema de Classificação da Função Motora Grossa (GMFCS). Rev Bras Fisioter. 2010;14(6):537-44. Disponível em: http://www.scielo.br/ pdf/rbfis/v14n6/a13v14n6.pdf.

16. Johnson RM. Guia dos símbolos de comunicação pictórica. Porto Alegre: CLIK; 1998.

17. Von Tetzchner S; Martinsen H. Introdução à comunicação aumentativa e alternativa. Portugal: Porto; 2000.

18. Ferreira-Donati GC, Deliberato D. Programa de educação familiar continuada em linguagem: contribuições de orientações escritas num modelo de educação à distância. In: Deliberato D, Gonçalves MJ, Macedo EC. Comunicação alternativa: teoria, prática, tecnologias e pesquisa. São Paulo: Memnon; 2009. p.302-11.

19. Goldoni NI. Orientação para familiares de alunos com paralisia cerebral usuários de sistemas de comunicação suplementar e alternativa [dissertação]. Marília (SP): Universidade Estadual Paulista; 2013 [citado 24 jan. 2015] Disponível em: http://www.marilia.unesp.br/Home/PosGraduacao/Educacao/Dissertacoes/goldoni_ni_me_mar. pdf.

20. Nunes LR. Modelos teóricos na comunicação alternativa e ampliada. In: Nunes LR. Favorecendo o desenvolvimento da comunicação em crianças e jovens com necessidades educacionais especiais. Rio de Janeiro: Dunya; 2003. p.15-47 [citado 24 fev. 2015]. Disponível em: http:// www.lateca-uerj.net/publicacoes/docs/Modelos $\% 20$ Te\%C3\%B3ricos\%20na\%20CAA\%2003.pdf.

21. Kent-Walsh J, Murzab K, Malanic M.D, Cathy Bingerd C. Effects of communication partner instruction on the communication of individuals using AAC: a meta-analysis. Augment Altern Commun. 2015;31(4):271-84. doi: 10.3109/07434618.2015.1052153.

22. Kent-Walsh J, McNaughton D. Communication partner instruction in AAC: present practices and future directions. Augment Altern Commun. 2005;21(3):195-204. http:// dx.doi.org/10.1080/07434610400006646.

Recebido em: 05.03.15

Aceito em: 06.07.16 